\title{
Les organisations rurales au cœur de la transformation des campagnes africaines?
}

Jean-Claude Deveze

\section{(2) OpenEdition}

\section{Journals}

Édition électronique

URL : http://journals.openedition.org/apad/3803

DOI : 10.4000/apad.3803

ISSN : 1950-6929

Éditeur

LIT Verlag

\section{Édition imprimée}

Date de publication : 1 décembre 1992

\section{Référence électronique}

Jean-Claude Deveze, "Les organisations rurales au cœur de la transformation des campagnes africaines ? », Bulletin de l'APAD [En ligne], 4 | 1992, mis en ligne le 26 juin 2008, consulté le 07 septembre 2020. URL : http://journals.openedition.org/apad/3803 ; DOI : https://doi.org/10.4000/ apad.3803

Ce document a été généré automatiquement le 7 septembre 2020.

Bulletin de l'APAD 


\title{
Les organisations rurales au cœur de la transformation des campagnes africaines?
}

\author{
Jean-Claude Deveze
}

1 Le rôle des "organisations rurales" ${ }^{1}$ (OR) est au cœur des interrogations sur les transformations des campagnes africaines. Au-delà des propos pessimistes portés sur les évolutions de l'agriculture africaine, il est incontestable que de multiples changements sont en cours sur le terrain, et en premier lieu que les OR se multiplient et se diversifient. Il est proposé d'essayer de s'y retrouver dans ce foisonnement, à partir de ce qui peut être appréhendé en Afrique francophone au Sud du Sahara et à Madagascar. Ceci nous amènera à poser des questions sur l'importance et la nature de ces organisations rurales, sur les rapports entre stratégies individuelles et collectives, sur les relations des OR avec leur environnement institutionnel, sur la professionnalisation de l'agriculture, etc.

Que recouvre le foisonnement des organisations rurales?

Diverses tentatives de typologie des OR ont été proposées selon leur origine, les fonctions qu'elles recouvrent, leur mode de fonctionnement, etc., mais il reste très difficile d'appréhender les conditions de leur émergence et de leur évolution -qui sont liées à des causes multiples dont certaines sont enracinées dans une histoire locale complexe.

3 Ce qui est à notre avis le plus important à considérer pour percevoir les évolutions et recompositions en cours, ce sont les facteurs de changement qui sont en œuvre.

Dans un contexte d'ajustement structurel et d'accentuation des déséquilibres des filières agricoles, le fait le plus nouveau est sans doute la remise en cause du rôle de l'Etat et des structures dépendant de lui. Des structures de développement ou d'aménagement ont été démantelées ou ont vu leurs missions recentrées sur des objets plus spécifiques. Cela a créé dans certains cas un vide qui est plus ou moins rapidement comblé par des OR. Le cas le plus significatif que nous connaissons est la multiplication des OR et l'apparition de structures fédératives dans le delta du fleuve Sénégal, à la 
suite du désengagement de l'Etat et de la réduction des fonctions confiées à la Société d'Aménagement et d'Equipement du Delta (SAED) ; cette dynamique a été favorisée par la mise au point du statut de Groupement d'Intérêt Economique ouvrant l'accès au crédit de la Caisse Nationale de Crédit Agricole, et par la distribution des terres par les communautés rurales, ce qui a permis de multiplier les aménagements privés.

5 Les initiatives les plus visibles proviennent souvent des sources de financement extérieures. Elles ont cherché à provoquer l'émergence des $\mathrm{OR}$, ou ont appuyé certaines d'entre elles, et elles privilégient de plus en plus le thème de la structuration du milieu rural en vue de favoriser un développement participatif. Il faut distinguer entre les bailleurs de fonds internationaux qui cherchent à définir leurs stratégies en ce domaine, et les ONG qui travaillent souvent à une échelle plus réduite. Il faut noter que le relatif désengagement, des bailleurs de fonds du secteur rural contribue à la remise en cause du rôle de l'Etat qui, faute de ressources, doit déléguer localement l'exercice de certaines fonctions.

6 Dans certains pays, un facteur nouveau d'organisation est la gestion des ressources naturelles par les villages. Ainsi, au Burkina, on commence à assister à la mise en place de comités de gestion des terroirs qui jouent un rôle grandissant dans l'adoption de règles permettant de trouver des solutions pour l'accueil des migrants, pour les parcours des animaux, pour la coupe des arbres, etc. Dans d'autres cas, on parle de développement local avec définition de règles communales.

7 L'élément le plus constant est la persistance d'impératifs économiques (organisation de la commercialisation et de l'approvisionnement, amélioration de l'accès au crédit, gestion des équipements, etc...) qui conduisent à la création et à la promotion d'OR. Ainsi, la réactivation de la filière café en Guinée a entrainé le lancement de 60 groupements. Cependant, les dynamiques économiques ne sont pas forcément durables ou maîtrisables, et l'on assiste à la fin de certains projets, ou lors de crises de certaines filières, à la remise en cause des structures paysannes mises en place. Ce fut particulièrement net dans le cas du Niger à la suite de la réduction de la production de l'arachide. Malheureusement, beaucoup d'organisations paysannes à but économique disparaissent aussi, faute d'une gestion sérieuse, d'une maîtrise des prestations, etc.

8 Il se produit aussi de multiples évolutions dans les rapports sociaux, compte tenu d'enjeux de pouvoir, d'enrichissement, etc., ce qui conduit à des changements d'attitude. Ainsi en zone cotonnière, les producteurs les plus performants, après avoir accepté le principe de caution solidaire du crédit, remettent en cause celui-ci dès que le taux de remboursement baisse (ce qui est de plus en plus le cas avec la baisse du prix du coton graine au producteur). Par contre, des femmes, des jeunes, prennent de plus en plus d'initiatives pour se faire reconnaître.

9 Les réalités les plus difficiles à appréhender recouvrent les facteurs culturels. Les OR s'insèrent au départ dans un tissu de communautés locales où les dimensions sociales et les préoccupations de maintien d'une certaine solidarité sont prioritaires. Ainsi au Mali, les associations villageoises en zone cotonnière ont permis l'utilisation des ristournes liées à la production du coton en faveur surtout des multiples besoins sociaux et culturels des villages. Dans certains cas, la dimension ethnique reste aussi très importante et est renforcée par l'alphabétisation en langue locale.

10 Quant aux interférences politiques, elles ont toujours existé. Ainsi, une des causes des difficultés de structuration du monde paysan après les indépendances a été, dans de nombreux pays, la politisation excessive d'un mouvement coopératif que l'Etat 
souhaitait contrôler au travers d'un parti unique. On entre dans une nouvelle phase, où théoriquement les progrès de la démocratisation dans un contexte libéral, devraient permettre l'acquisition par les OR d'une réelle autonomie par rapport à l'Etat et aux forces qui le dirigent. En fait, les tentatives de récupération politique se perpétuent sous des formes plus complexes. Ainsi dans plusieurs pays d'Afrique de l'Ouest, Etat et paysans "cravatés" cherchent à canaliser le mouvement paysan au travers de chambres d'agriculture rénovées.

11 Ce sont la plupart du temps une conjonction de facteurs et d'impératifs qui poussent les paysans à s'organiser sur le plan local. Ainsi en Basse Casamance, l'intervention de projets et d'ONG, les nécessités économiques à la suit y d'une dégradation de l'écologie, les initiatives de leaders, le désengagement de l'Etat, ont conduit à la création, en 1988 à Bignona, d'une Coordination des Organisations Rurales du Département (CORD) ${ }^{2}$.

Il est intéressant de disposer d'analyses croisées des évolutions en cours provenant des acteurs locaux concernés et des observateurs externes au milieu. Une tentative de ce type est en cours au Mali ${ }^{3}$.

Comment s'articulent les stratégies individuelles et collectives?

Pour expliquer les dynamiques de création d'OR, la dimension "intérêts collectifs" a été souvent privilégiée; mais il ne faut pas sous-estimer les stratégies individuelles des personnes influentes et des notables. Un des facteurs importants de remise en cause des stratégies adoptées par les communautés villageoises est le poids grandissant des néoruraux: fonctionnaires licenciés, commerçants investissant dans l'agriculture, diplômés en recherche d'emploi, petits entrepreneurs, etc. Ils cherchent à s'insérer dans le tissu des OR et, dans certains cas, ils montent leurs propres organisations grâce à divers appuis.

De même, certains chefs de famille essaient de créer des groupements permettant de défendre les intérêts familiaux ou individuels.

Cependant se pose souvent le problème de dégager des consensus entre des stratégies individuelles. Autrefois, le palabre au sein des communautés rurales était un des instruments privilégiés de régulation sociale. Avec l'émergence des néoruraux, l'arrivée des migrants, la diversification des stratégies individuelles, un nouveau lieu d'élaboration de consensus peut être l'OR, dans la mesure où des compromis entre stratégies individuelles et collectives y sont trouvés. Dans le cas du delta du fleuve Sénégal, il semble qu'il y ait plutôt une synergie positive entre celles-ci, les organisations fédératives apportant des appuis sur les plans techniques et fonciers aux petits groupements familiaux, et ces derniers donnant du poids aux organisations fédératives auxquelles ils adhèrent pour défendre leurs intérêts ${ }^{4}$.

Dans de nombreux cas, les groupements végètent et se disloquent à la suite de divergences entre des tenants de diverses stratégies individuelles. Ainsi, ceux qui peuvent rembourser leurs redevances liées à l'irrigation préfèrent fonder entre eux de nouveaux groupements et accroître leurs superficies rizicoles dans le Nord Cameroun.

On peut se demander en fin de compte si le plus important n'est pas la nature des stratégies en cause. Dans la mesure où des dynamiques économiques liées à des filières de production existent, il est possible aux individus et aux OR de soutenir des stratégies offensives, ce qui rend plus facile les synergies de stratégies entre les plus entreprenants, mais risque aussi de détruire le tissu social et certaines solidarités.

Vers un mouvement paysan et/ou vers une profession agricole? 

ou sur la constitution d'une profession agricole. Dominique Gentil et Marie-Rose Mercoiret ${ }^{5}$ ont proposé cinq critères pour tenter d'apprécier l'existence de mouvements paysans :

- autonomie intellectuelle et financière

- objectifs conscients et explicites

- rapports significatifs avec l'Etat et/ou le reste de la société civile

- taille ou poids économique/politique suffisant

- organisation interne déjà établie. agricole capable de les aider à approfondir les mutations en cours ${ }^{6} \mathrm{du}$ fait de multiples objectifs interdépendants: recherche d'une structuration efficiente du monde rural, appui aux initiatives individuelles et collectives, redéfinition de la place de l'agriculture dans le développement rural, émergence de partenaires professionnels capables de négocier la politique agricole, etc...

Les organisations rurales comme acteurs dans la mise en œuvre des interventions en milieu rural?

$\mathrm{Au}$ cœur des incertitudes sur l'avenir du monde rural en Afrique au Sud du Sahara se pose la question de l'avenir des institutions partenaires des paysans africains et malgaches. Il suffit de prendre quelques exemples pour mettre en évidence les difficultés de la période actuelle de transition :

- les grands périmètres irrigués ont été placés sous la tutelle de sociétés d'aménagement qui exerçaient aussi des fonctions de développement rural (vulgarisation, approvisionnement, crédit, commercialisation primaire, transformation, régulation foncière, etc). La remise en cause de ces sociétés ou même leur disparition (cas de la société d'aménagement du lac 
Alaotra à Madagascar) devrait conduire_à une nouvelle répartition des tâches entre des acteurs qui ont du mal à se redéfinir ou à s'organiser : État, société de gestion de l'eau, communautés rurales, organisations agricoles ou d'usagers, privés, etc ;

- les zones cotonnières ont été en Afrique francophone confiées pour la plupart à des sociétés agro-industrielles qui exerçaient des fonctions non seulement d'appui aux producteurs (vulgarisation, approvisionnement, crédit), mais aussi de développement régional (organisation des villages, entretien des pistes, appui à la formation des producteurs, etc). La crise liée à la baisse des cours du coton et les multiples remises en question ${ }^{7}$ de ce type de développement intégré conduisent à s'interroger sur l'évolution des diverses institutions concernées, et en particulier sur le rôle futur des sociétés "cotonnières" ;

- les zones forestières humides ont été durant longtemps placées sous la "protection" de l'administration forestière qui attribuait par ailleurs des permis de coupe aux exploitants forestiers. Les pressions des écologistes, désireux de protéger ces ressources naturelles, et les dénonciations de la mauvaise gestion de ce patrimoine par les forestiers, reposent la question des acteurs qui devraient être responsables des forêts restantes et des surfaces reboisées. l'atteinte d'objectifs qu'elles avaient définis. Ainsi le Comité d'Action pour le Développement du Fogny en Sud Casamance négocie avec des sources de financement extérieures l'attribution des moyens nécessaires à ses programmes de développement local.

31 Le plus difficile à mettre au point est le rôle que les OR devraient être amenées à jouer comme partenaire dans la régulation des divers processus engagés: gestion des aménagements et des ressources naturelles, appropriation du foncier, distribution du crédit, développement local, mise en place de circuits d'approvisionnement ou de commercialisation, etc.

Que manque-t-il aux organisations rurales pour aller plus loin dans leur contribution au développement? 

actuelle de nombreuses OR est assez inquiétante sur de nombreux points :

- forte dépendance vis-à-vis des notables et leaders, et des personnes ou structures qui leur procurent des financements et des appuis ;

- temps de maturation souvent trop court, du fait des multiples incitations à s'engager dans de nouvelles voies à la suite du désengagement de l'État, de la redistribution des rôles et fonctions, des incitations extérieures, etc. ;

- difficultés de gestion et d'organisation, accentuées dans certains cas par le manque de cadre juridique ${ }^{8}$ ou d'organismes de contrôle et d'arbitrage ;

- autorités ambiguës, souvent officiellement favorables aux OR, mais souhaitant garder leur pouvoir sur elles ;

- manque d'informations à leur disposition sur le contexte dans lequel elles doivent trouver leur voie ;

- tendance à davantage prendre en compte les relations sociales dans le court terme, qu'à gérer dans la durée les intérêts collectifs (ainsi l'entretien de périmètres irrigués est souvent négligé par un groupement qui préfère diminuer les redevances ou les consacrer à d'autres objets) ;

- mauvais remboursement des crédits obtenus.

D'autre part, on trouve de multiples éléments expliquant les évolutions positives constatées :

- importance de la formation des responsables (école ou alphabétisation fonctionnelle, voyages à l'extérieur, confrontations avec le pouvoir, etc.) ;

- présence de certaines dynamiques économiques et/ou de contraintes sévères obligeant à s'organiser ;

- réactivation de solidarités locales ;

- collaborations avec des structures de développement du pays ou des organismes d'appui respectueux de leurs démarches ;

- statuts juridiques simples à utiliser ;

- contexte politique permettant le débat

En dernier ressort, il apparaît que ce qui manque le plus aux OR pour s'imposer, c'est du temps. Il subsiste de forts risques pour que les partenaires extérieurs (sources de financement, Etat, ONG, etc.) cherchent à faire passer leurs vues en mettant en place des projets, appuis ou interventions qui n'ont pas été conçus ou appropriés par les OR. Il s'agit donc d'appuyer sans étouffer, d'initier sans imposer, d'accompagner sans précipiter afin de construire sur des bases solides. La confrontation avec l'extérieur présente l'avantage d'introduire des facteurs de changement, mais aussi l'inconvénient de troubler le jeu si les OR ne maîtrisent pas les choix qui s'offrent à elles.

Dans ce domaine complexe, le rôle de la coopération internationale devrait donc être réexaminé avec prudence, en tenant compte des renforcements en cours du cadre démocratique. Le recours à des sociologues et des anthropologues devraient être accru pour éclairer les décisions d'appui.

Répondre aux questions que pose l'avenir des OR reste difficile, compte tenu des incertitudes sur les transformations en cours des campagnes africaines. Selon le point de vue privilégié par les partenaires de ces $\mathrm{OR}$, les hypothèses de travail sont différentes. 


\section{NOTES}

1.Le terme "organisation rurale" est plus large que "organisation paysanne". Il permet d'inclure les néoruraux, les artisans ; d'englober les approches en termes de développement local.

2.Voir l'article de Jacques Berthomé dans le $n^{\circ} 10$ de la lettre du réseau GAO.

3.Ébauche de synthèse et quelques entretiens sur les organisations paysannes du Mali. Gaudens agron. Réseau GAO-FPH - 9 juin 1992.

4.Voir "Aperçus sur les stratégies des producteurs et des Organisations paysannes dans le Delta du Fleuve Sénégal", J. M. Yung \& Zaslasky - CCCE - Mars 1992.

5.Y -a-t-il un mouvement paysan en Afrique Noire ? - Revue Tiers-Monde - $\mathrm{n}^{\circ} 128$ IEDES, Oct-Déc. 1991.

6.Voir J-P. Prudhomme - n¹0 de la lettre du réseau GAO - 4ème trimestre 1991 - 1er trimestre 1992. 
7.Séparation des actions de développement et des activités agro-industrielles, création de services agricoles séparés, souci de promouvoir une fonction animation-formation autonome, etc.

8.Il est plus facile au Mali de faire reconnaitre un parti politique qu'une organisation rurale.

\section{AUTEUR}

JEAN-CLAUDE DEVEZE

CCCE et GAO 